\title{
Nanobody conjugated PLGA nanoparticles for active targeting of African Trypanosomiasis
}

\author{
José L. Arias a , Juan D. Unciti-Broceta b,c,d , José Maceira ${ }^{\text {b,c,d }}$, Teresa del Castillo ${ }^{\text {b,c,d }}$, José Hernández-Quero ${ }^{\text {b }}$, \\ Stefan Magez ${ }^{\text {e,f }}$, Miguel Soriano ${ }^{\text {d,g,1 }}$, José A. García-Salcedo b,c,d,*,1 \\ a Departamento de Farmacia y Tecnología Farmacéutica, Facultad de Farmacia, Universidad de Granada, Granada, Spain \\ b Unidad de Enfermedades Infecciosas y Microbiología, Instituto de Investigación Biosanitaria ibs.GRANADA, Hospitales Universitarios de Granada/Universidad de Granada, Granada, Spain \\ " Instituto de Parasitología y Biomedicina "López-Neyra" (IPBLN-CSIC), PTS Granada, Armilla, Spain \\ ' GENYO, Centro de Genómica e Investigación Oncológica: Pfizer/Universidad de Granada/Junta de Andalucía, PTS Granada, Granada, Spain \\ e Unit of Cellular and Molecular Immunology, Vrije Universiteit Brussel, Brussels, Belgium \\ ${ }^{\mathrm{f}}$ Department of Structural Biology, VIB, Vrije Universiteit Brussel, Brussels, Belgium \\ ' Departamento de Agronomía, Universidad de Almería, Almería, Spain
}

\section{A R T I C L E I N F O}

\section{Article history:}

Received 14 July 2014

Accepted 3 November 2014

Available online 10 November 2014

\section{Keywords:}

Polymeric nanoparticles

PLGA

Nanobody nanoparticles conjugation

PEGylation

Specific cell targeting

Human African trypanosomiasis

\begin{abstract}
A B S T R A C T
Targeted delivery of therapeutics is an alternative approach for the selective treatment of infectious diseases. The surface of African trypanosomes, the causative agents of African trypanosomiasis, is covered by a surface coat consisting of a single variant surface glycoprotein, termed VSG. This coat is recycled by endocytosis at a very high speed, making the trypanosome surface an excellent target for the delivery of trypanocidal drugs. Here, we report the design of a drug nanocarrier based on poly ethylen glycol (PEG) covalently attached (PEGylated) to poly(D,L-lactide-co-glycolide acid) (PLGA) to generate PEGylated PLGA nanoparticles. This nanocarrier was coupled to a single domain heavy chain antibody fragment (nanobody) that specifically recognizes the surface of the protozoan pathogen Trypanosoma brucei. Nanoparticles were loaded with pentamidine, the first-line drug for T. b. gambiense acute infection. An in vitro effectiveness assay showed a 7-fold decrease in the half-inhibitory concentration $\left(\mathrm{IC}_{50}\right)$ of the formulation relative to free drug. Furthermore, in vivo therapy using a murine model of African trypanosomiasis demonstrated that the formulation cured all infected mice at a 10 -fold lower dose than the minimal full curative dose of free pentamidine and $60 \%$ of mice at a 100 -fold lower dose. This nanocarrier has been designed with components approved for use in humans and loaded with a drug that is currently in use to treat the disease. Moreover, this flexible nanobody-based system can be adapted to load any compound, opening a range of new potential therapies with application to other diseases.
\end{abstract}

(c) 2014 Elsevier B.V. All rights reserved.

\section{Introduction}

African trypanosomiasis is a disease with a devastating socioeconomic impact in sub-Saharan Africa. The causative agent, Trypanosoma brucei $s s p$, is transmitted by the bite of and infected fly of the genus Glossina to humans or domestic livesock [1-3]. Human African trypanosomiasis (HAT), also known as sleeping sickness, is caused by T. $b$. gambiense or $T$. $b$. rhodesiense and is typically fatal without chemotherapy. HAT is endemic in 36 African countries and around 60 million people are at risk of being infected. The prevalence has been variable during the twentieth century coinciding the re-emergence of the number of cases with periods of famine and war [4,5]. The public health situation has improved recently with surveillance and control efforts averting more than

\footnotetext{
* Corresponding author at: GENYO, Centro de Genómica e Investigación Oncológica: Pfizer/Universidad de Granada/Junta de Andalucía, PTS Granada Avda. de la Ilustración 114, 18016 Granada, Spain. Tel.: + 349687155 00x165; fax: + 34958637071 .

E-mail address: jags@genyo.es (J.A. García-Salcedo).

1 Both authors contributed equally.
}

1.6 millions disability-adjusted life years in 2004. However, displacement of populations, conflicts, and poverty may lead to increased transmission, with severe social and economic consequences.

African trypanosomes are extracellular parasites transmitted by the bite of tsetse flies. Trypanosomes are able to evade the host immune system by changing the Variant Surface Glycoprotein (VSG), in a process termed antigenic variation [6,7]. The VSG is organized as densely packed dimers and works as a physical barrier impeding antibody recognition of invariant inner epitopes (Fig. 1). The rate of parasite surface turnover is very high and occurs in the flagellar pocket, which is an invagination of the plasma membrane around the base of the flagellum and the sole site for endocytosis [8]. This is a defence mechanism to degrade host antibodies bound to the surface of the parasite $[9,10]$ but is also important for the uptake of some essential nutrients from the host blood, such as transferrin and lipoproteins [11,12].

Antigenic variation eliminates the possibility of developing an effective vaccine, leaving chemotherapy as the only method to fight against HAT disease. However, the drugs currently in use to treat 
A VSG Structure

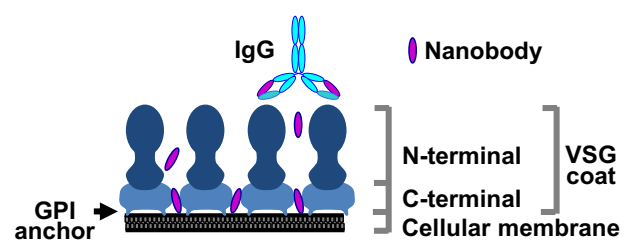

B

NbAn33-pentamidine PEGylated PLGA NP

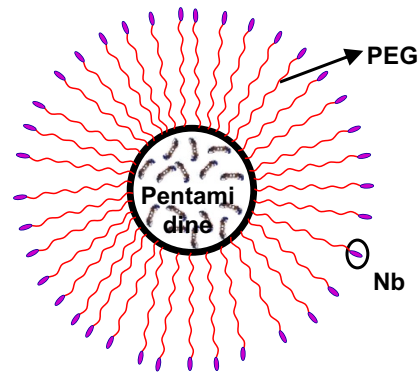

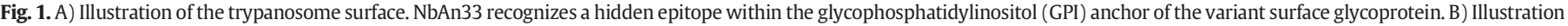
of a NbAn33-pentamidine-PLGA NP.

HAT are old and very limited, with most having serious side effects, including encephalopathy, toxicity and death $[13,14]$. Currently, there are only five licensed drugs for the treatment of HAT. Pentamidine and suramin are suitable for treating the disease before parasites invade the central nervous system; pentamidine is the drug of choice in the treatment of first-stage T. b. gambiense HAT, and suramin is used for first-stage T. b. rhodesiense HAT [13,14]. Melarsoprol, eflornithine and eflornithine in combination with nifurtimox are the available treatments for advanced disease. Clearly, alternative therapeutic strategies are needed.

Nowadays, there are two approaches to develop new therapies: one is the search for new drugs and the other is the optimisation of actual formulations and their applications [15]. Design of nanoscale devices for drug-delivery is one of the most important goals in medicine and pharmaceutical technology [16]. Different systems based on nanoparticles (NPs) have been developed, including inorganic, magnetic and polymeric NPs [17]. These systems have many advantages when compared with conventional therapies. They protect drugs against oxidoreduction and enzymatic reactions, increasing the bioavailability and reducing the effective doses and negative side effects [17]. Incorporation of poly ethylene glycol (PEG) molecules on their surface (PEGylation) increases nanoparticle circulation times by reducing liver uptake. Nanoparticle PEGylation also provides moieties to attach biofunctional molecules for specific cell or organ targeting, such as antibodies [18,19].

Poly (lactic-co-glycolic acid) (PLGA) is a versatile polymer that is widely used for drug encapsulation through the formulation of NPs. PLGA is biodegradable and biocompatible and its use in humans for parenteral administration has been approved by the Food and Drug Administration and the European Medicine Agency [20]. The formulations and methods of PLGA NPs synthesis are well standardized and the NPs are adaptable to different types of drugs and administration routes. Moreover, their biological interaction with the environment and the rate of drug release can be modulated by modifying their physicochemical properties like shape, surface charge and hydrophobicity [21-23].

Nanobodies are single-domain antibody fragments derived from functional heavy-chain antibodies (HCAbs) of camelids [24-27] (Fig. 2). Unlike conventional antibodies, which are constituted of two identical heavy-chains and two identical light-chains, heavy-chain antibodies from camelids have lost the light-chains (Fig. 2). The heavy chain of HCAbs is composed of three instead of four globular domains: two constant domains $\left(\mathrm{C}_{\mathrm{H}} 2-\mathrm{C}_{\mathrm{H}} 3\right)$ with high homology to conventional antibodies and one variable domain $[28,29]$. The $\mathrm{C}_{\mathrm{H}} 1$ domain of conventional antibodies is lost. The variable domain (nanobody), can be cloned and expressed through recombinant gene technology, and is fully functional [30].
Nanobodies have several advantageous properties compared to conventional antibodies: i) small size ( $15 \mathrm{kDa}$ ), which is suitable for targeting epitopes in obstructed locations; ii) high affinity and specificity; iii) high stability and solubility; iv) not immunogenic to animals or humans; and v) well produced in bacteria and yeasts at low manufacturing costs [31]. Nanobodies have been successfully used for specific targeting in different therapeutic approaches which comprise direct blocking of receptors or viruses [32,33], chimeric fusion proteins [34-36], and conjugation to drug carriers [31,37-39]. Nanobodies have proven to be efficient for the treatment of infectious protozoan diseases, such as African trypanosomiasis [34] and malaria [40].

In the current study, we have taken advantage of the highly active endocytosis process to specifically and effectively deliver drugs into this protozoan parasite. The aim of this work was to develop a new polyvalent drug delivery system for the treatment of African trypanosomiasis based on PLGA NPs conjugated with a nanobody that specifically recognizes conserved cryptic epitopes on the parasite surface.

\section{Materials and methods}

\subsection{Chemicals}

Water used in the experiments was deionized and filtered with a Milli-Q Academic System (Millipore, Saint Quentin-en-Yvelines,

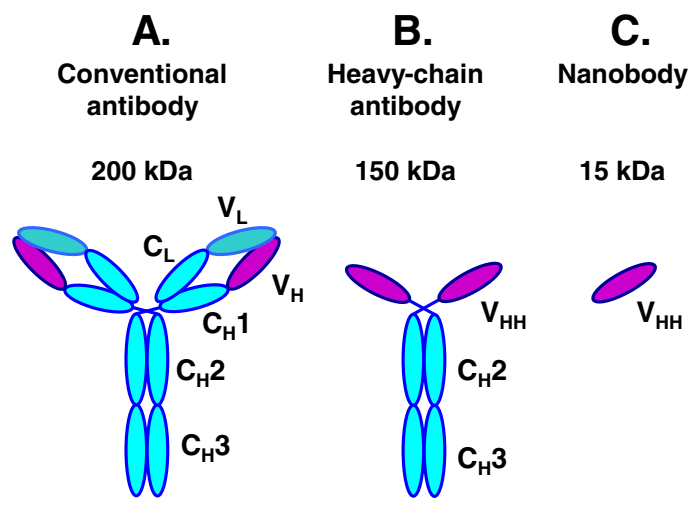

Fig. 2. Scheme of a conventional IgG antibody. (A), a camelid heavy-chain IgG antibody (B) and the variable domain of heavy chain antibodies, $\mathrm{VHH}$, also known as a nanobody (C). Conventional antibodies have two identical heavy chains, formed by $V_{H}, C_{H} 1, C_{H} 2$ and $\mathrm{C}_{\mathrm{H}} 3$ domains and two identical light chains, $\mathrm{V}_{\mathrm{L}}$ and $\mathrm{C}_{\mathrm{L}}$ domains. Heavy-chain antibodies contain a single $V_{H H}$ and two constant domains $\left(C_{H} 2\right.$ and $\left.C_{H} 3\right)$. A Nanobody is the $V_{H H}$ domain of heavy-chain antibodies obtained by recombinant gene technology. $C_{H} 1-3$ : constant domains of heavy chain. $\mathrm{C}_{\mathrm{L}}$ : constant domain of light chain. $\mathrm{V}_{\mathrm{H}}$ : variable domain of heavy chain. $\mathrm{V}_{\mathrm{L}}$ : variable domain of light chain. 
France). All chemicals used were of analytical quality from Panreac (Barcelona, Spain), except for PLGA 50:50 [molecular weight $\left(\mathrm{M}_{\mathrm{w}}\right)$ : $12000 \mathrm{Da}$; inherent viscosity: $0.24 \mathrm{dL} / \mathrm{g}$ ], dextran-70, polyvinyl alcohol (PVA), $\mathrm{NH}_{2}$-PEG-COOH ( $\mathrm{M}_{\mathrm{w}}$ : $3 \mathrm{kDa}$ ), 1-ethyl-3-(3-dimethylaminopropyl) carbodiimide (EDC), and $\mathrm{N}$-hydroxysuccinimide (NHS) from Sigma-Aldrich (Munich, Germany).

\subsection{Nanobody purification}

The NbAn33 was expressed and purified as described previously [41]. Briefly, NbAn33 incorporating the pelB export signal and a sixhistidine-tag was expressed under the Lac promoter in Escherichia coli WK6 cells (European Collection of Cell Culture). The nanobody was purified from periplasmic extracts as described earlier [42]. The six-histidine-tagged NbAn33 was purified using a Ni-NTA column (Qiagen, Hilden, Germany), followed by gel filtration on a Superdex column (Amersham Biosciences, Uppsala, Sweden).

\subsection{Synthesis of pentamidine-loaded NbAn33-coated PEGylated PLGA NPS (NbAn33-pentamidine-PLGA NPS)}

PLGA NPs were synthesized by a water-in-oil-in-water (w/o/w) double emulsion/solvent evaporation (DE/SEV) methodology [43-45]. Briefly, $3 \mathrm{~mL}$ of an aqueous solution of dextran-70 (1\%, w/v) was added to $10 \mathrm{~mL}$ of a PLGA solution in ethyl acetate $(2.5 \%, w / v)$, under mechanical stirring $(20,000 \mathrm{rpm})$. The resulting $\mathrm{w} / \mathrm{o}$ emulsion was poured under mechanical stirring $(20,000 \mathrm{rpm})$ into $50 \mathrm{~mL}$ of a $0.3 \%(w / v)$ PVA aqueous solution. Stirring was continued for $15 \mathrm{~min}$. As a result, a w/o/w emulsion was formed, which was then diluted in $100 \mathrm{~mL}$ of a $2 \%(w / v)$ PVA aqueous solution. Finally, mechanical stirring (1000 rpm, $\left.25^{\circ} \mathrm{C}\right)$ was continued during $12 \mathrm{~h}$ to completely evaporate the organic solvent. The colloidal suspension was then subjected to a cleaning procedure that included repeated cycles of centrifugation (30 min at 11,000 rpm, Centrikon T-124 high-speed centrifuge, Kontron, Zürich, Switzerland) and re-dispersion in water, until the conductivity of the supernatant was $\leq 10 \mu \mathrm{S} / \mathrm{cm}$.

Standard EDC/NHS chemistry was used to link the heterofunctional PEG chains ( $3 \mathrm{kDa}, \mathrm{NH}_{2}$-PEG-COOH) and the activated carboxyl groups of the PLGA surface [19]. Carboxyl groups were activated in $5 \mathrm{~mL}$ of isotonic $0.1 \mathrm{M} 2$-( $\mathrm{N}$-morpholino) ethanesulphonic acid saline (MES) buffer ( $\mathrm{pH} \mathrm{5.5)}$ ) for $1 \mathrm{~h}$ at room temperature with EDC and NHS (2:1.2 weight ratio). The "activated" PLGA NPs dispersion was then centrifuged $(11,000 \mathrm{rpm}, 30 \mathrm{~min})$ and re-suspended in $3 \mathrm{~mL}$ of phosphate buffered saline (PBS, pH 7.4). The reaction of PLGA NPs with $\mathrm{HCl} \cdot \mathrm{NH}_{2}$-PEG-COOH (1.3:1 weight ratio) was accomplished at room temperature for $12 \mathrm{~h}$. The resulting PEGylated (PEG-b-PLGA) NPs were then centrifuged $(11,000 \mathrm{rpm}, 30 \mathrm{~min})$ and washed with PBS ( $\mathrm{pH} 7.4$ ) to remove the unbound PEG.

The nanobody was coupled to the PEGylated PLGA NPs (PLGA NPs) via the EDC/NHS chemistry. Briefly, a nanobody aqueous solution $(1 \mathrm{mg} / \mathrm{mL})$ was added to the NP suspension (10:1 weight ratio) and the system was allowed to react during $3 \mathrm{~h}$ under mechanical stirring (200 rpm, room temperature). After that, $10 \mathrm{~mL}$ of PBS (pH 7.4) were added, and the NP dispersion was centrifuged (11,000 rpm, $30 \mathrm{~min}$ ). Finally, fluorescent NPs to be used in the cell uptake experiments were synthesized using 5(6)-amino fluorescein (1:10 equivalent ratio fluorescein: carboxyl groups) following the same chemistry.

The entrapment procedure for pentamidine loading was analogous to that described for the preparation of the drug-unloaded NPs except that the aqueous solution of dextran-70 further contained increasing concentrations of pentamidine (up to $0.01 \mathrm{M}$ ). As a result, pentamidineloaded PEGylated PLGA NPs (pentamidine-PLGA NPs), and NbAn33pentamidine-PLGA NPs were obtained. The production performance (yield, \%) of all the formulation conditions was also determined [ (amount of NPs obtained $(\mathrm{mg}) / \mathrm{sum}$ of materials used in the preparation of the $\mathrm{NPs}(\mathrm{mg})) \times 100]$.

\subsection{Characterization methods}

Photon correlation spectroscopy (PCS, Malvern Autosizer ${ }^{\circledR}$ 4700, Malvern Instruments S.A., Worcestershire, UK) was used to determine in triplicate the mean particle size ( \pm standard deviation) of all the polymeric particles at $25^{\circ} \mathrm{C}$. To that aim, the scattering angle was set at $60^{\circ}$, and the measurement was done after an appropriate dilution of the aqueous nanoparticulate dispersions $(0.1 \%, w / v)$. In addition, the stability of the nanoformulations was evaluated by measuring the size of the particles after 1 month of storage in water at $4{ }^{\circ} \mathrm{C}$.

PEGylation efficiency of the PLGA NPs was qualitatively determined by ${ }^{1} \mathrm{H}$ nuclear magnetic resonance $\left({ }^{1} \mathrm{H}-\mathrm{NMR}\right.$, Bruker AM-300, Bruker Corporation, Billerica, USA). ${ }^{1} \mathrm{H}-\mathrm{NMR}$ data was obtained using deuterated dimethyl sulfoxide (d6-DMSO) as solvent, and the most significant bands of PEG and PLGA were identified. Chemical shifts were measured in parts per million (ppm) using tetramethylsilane (TMS) as an internal reference. In addition, the grafting density of PEG over the poly(D, L-lactide) (PLA) backbone of the copolymer was calculated integrating the peak of PEG (3.5 ppm) and PLGA (4.8 ppm) in the 1H-NMR spectra.

Drug concentration in all the nanoparticulate formulations was measured by ultraviolet-visible (UV-Vis) absorption measurements (at the maximum absorbance wavelength: $261 \mathrm{~nm}, 8500 \mathrm{UV}-\mathrm{Vis}$ Dinko spectrophotometer, Dinko Instruments, Barcelona, Spain), using quartz cells of $1 \mathrm{~cm}$ path length. The spectrophotometric method employed was previously validated by ultra-high-performance liquid chromatography-tandem mass spectrometry (UPLC-MS/MS) and verified for accuracy, precision, and linearity. Drug loading measurements were done in triplicate by UV-Vis spectrophotometric quantification of the pentamidine remaining in the supernatant after NP centrifugation $\left(11,000 \mathrm{rpm}, 30 \mathrm{~min}, 25^{\circ} \mathrm{C}\right)$. Drug incorporation was expressed in terms of pentamidine entrapment efficiency (\%) [(mass of drug incorporated $(\mathrm{mg}) /$ total mass of drug used in the loading experiments $(\mathrm{mg})) \times 100$ ], and pentamidine loading $(\%)$ [(mass of drug incorporated $(\mathrm{mg}) /$ mass of NPs obtained $(\mathrm{mg})) \times 100]$.

Similarly, it was investigated the density of NbAn33 on the surface of PLGA NPs. The procedure consisted in UV-Vis spectrophotometric measurements of the nanobody remaining in the supernatant after NP centrifugation $\left(11,000 \mathrm{rpm}, 30 \mathrm{~min}, 25^{\circ} \mathrm{C}\right)$. The method was previously validated by UPLC-MS/MS.

The electrokinetic characterization of blank (drug unloaded) NPs and drug-loaded NPs was used to confirm the type of pentamidine loading (surface adsorption, or absorption into the NPs). Zeta potential $(\zeta$ ) determinations (Malvern Zetasizer 2000 electrophoresis device, Malvern Instruments S.A., Worcestershire, UK) were performed at $25^{\circ} \mathrm{C}$, after $24 \mathrm{~h}$ of contact of the particles in water $(\approx 0.1 \% \mathrm{w} / \mathrm{v}$, at pH 5 and $1 \mathrm{mM} \mathrm{KNO}_{3}$, and under mechanical stirring: $50 \mathrm{rpm}$ ).

Pentamidine release experiments were performed in triplicate following the dialysis bag method using the NbAn33-pentaamidinePLGA NPs with the greater drug entrapment efficiency values $(\approx 72 \%$, see Table 1 ). To that aim, the dialysis bags were soaked in water for $12 \mathrm{~h}$ before use. Bags with a cut-off of $2000 \mathrm{Da}$ (Spectra/Por ${ }^{\circledR} 6$ dialysis membrane tubing, Spectrum ${ }^{\circledR}$ Laboratories, New Jersey, USA) retained the NPs and allowed the free pentamidine molecules to diffuse into the release media. The release media was PBS at $\mathrm{pH} 7.4$ or $\mathrm{pH} 5.0$ at $37^{\circ} \mathrm{C}$. Samples of $1 \mathrm{~mL}$ of NP dispersion $(0.55 \mathrm{mg} / \mathrm{mL}$ of drug) were poured into the bag with the two ends fixed by clamps. Then, the bag was placed in a conical flask filled with $150 \mathrm{~mL}$ of PBS, and was mechanically stirred at $200 \mathrm{rpm} .1 \mathrm{~mL}$ samples of the medium were withdrawn at different time intervals $(0.5,1,3,6,9,12$, and $24 \mathrm{~h}$, and $2,3,4,5,6,7$, $8,9,10,11$, and 12 days) for UV-Vis spectrophotometric analysis $(261 \mathrm{~nm})$. An equal volume of release medium, maintained at the same temperature, was added after sampling to ensure sink conditions. Furthermore, pentamidine release experiments were done under the same experimental conditions used to coat pentamidine-PLGA NPs with the nanobody $\left(3 \mathrm{~h}, 25^{\circ} \mathrm{C}\right.$, and mechanical stirring: $\left.200 \mathrm{rpm}\right)$. In 
Table 1

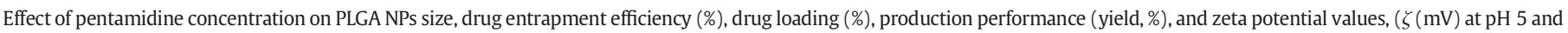
$1 \mathrm{mM} \mathrm{KNO}_{3}$ ).

\begin{tabular}{|c|c|c|c|c|c|}
\hline [Pentamidine] (M) & Size $(\mathrm{nm})$ & Pentamidine entrapment efficiency (\%) & Pentamidine loading (\%) & Production performance (\%) & $\zeta(\mathrm{mV})$ \\
\hline 0 & $142 \pm 21$ & - & - & $97 \pm 1$ & $-23 \pm 4$ \\
\hline $5 \times 10^{-5}$ & $131 \pm 29$ & $27 \pm 4$ & $0.00552 \pm 0.00082$ & $95 \pm 2$ & $-25 \pm 3$ \\
\hline $10^{-4}$ & $140 \pm 22$ & $41 \pm 3$ & $0.01675 \pm 0.00122$ & $96 \pm 3$ & $-22 \pm 3$ \\
\hline $5 \times 10^{-4}$ & $146 \pm 32$ & $52 \pm 4$ & $0.10622 \pm 0.00817$ & $97 \pm 2$ & $-23 \pm 3$ \\
\hline $10^{-3}$ & $150 \pm 28$ & $59 \pm 3$ & $0.24102 \pm 0.01225$ & $95 \pm 1$ & $-25 \pm 4$ \\
\hline $5 \times 10^{-3}$ & $138 \pm 27$ & $64 \pm 4$ & $1.30721 \pm 0.08171$ & $96 \pm 2$ & $-24 \pm 4$ \\
\hline $10^{-2}$ & $145 \pm 35$ & $72 \pm 6$ & $2.94123 \pm 0.24511$ & $97 \pm 1$ & $-22 \pm 3$ \\
\hline
\end{tabular}

this case, the aim was to clarify if drug release from NPs further occurred during the NbAn33 coating procedure.

\subsection{Trypanosomes and growth conditions}

Monomorphic bloodstream T. brucei, strain AnTat 1.1 (Institute of Tropical Medicine, Antwerpen, Belgium) was cultivated in hydrargyrum medium-arc iodide-9 (HMI-9) medium supplemented with $20 \%$ heatinactivated foetal bovine serum (Sigma-Aldrich, Munich, Germany), at $37^{\circ} \mathrm{C}$ in an atmosphere of $5 \% \mathrm{CO}_{2}$.

\subsection{In vitro viability assay}

Cell viability was evaluated as previously described [46]. Eleven dilution points were tested, ranging from $680 \mu \mathrm{M}$ to $165 \mathrm{pM}$ in pentamidine concentration. The plates were read on an Infinite ${ }^{\circledR}$ F200 PRO multimode reader (Tecan Group Ltd., Männedorf, Switzerland) using an excitation wavelength of $535 \mathrm{~nm}$ and an emission wavelength of $590 \mathrm{~nm}$. Data are the average of six determinations per concentration, repeated thrice. The half-inhibitory concentration $\left(\mathrm{IC}_{50}\right)$ value was calculated (GraphPad Prism 5 software, GraphPad Software, Inc., La Jolla, USA) as the drug concentration required to reduce the fluorescence output of the controls by $50 \%$.

\subsection{In vivo therapy experiment}

Five C57BL/6J female mice (8-week-old, Jackson Laboratories, Bar Harbor, USA) per group, and monomorphic AnTat $1.1 \mathrm{~T}$. brucei were used in this study. Mouse infection was obtained by intraperitoneal (i.p.) injection of PBS $(100 \mu \mathrm{L})$ containing $10^{4}$ parasites. The number of parasites in blood was estimated using a Neubauer counting chamber (Laboroptik Co., Bad Hamburg, Germany) and a light microscope (Leica Microsystems GmbH, Wetzlar Germany). The treatment was administrated i.p. on days 3 (when the parasitemia was detectable), 7 , 11 , and 15 post infection, in a daily dose (volume: $200 \mu \mathrm{L} /$ dose). Pentamidine doses were $0.025,0.25$, or $2.5 \mathrm{mg} \mathrm{kg}^{-1}$ of free pentamidine or pentamidine loaded into the NPs. The control group was treated with saline serum $(\mathrm{NaCl} 0.9 \%)$. Animals were humanely sacrificed when exhibiting severe disease symptoms, or if the parasitemia was $\geq 10^{8}$ parasites $/ \mathrm{mL}$.

\subsection{Field emission high resolution scanning electron microscopy}

T. brucei bloodstream forms $\left(10^{7}\right)$ were incubated $\left(10 \mathrm{~min}, 37^{\circ} \mathrm{C}\right)$ in $1 \mathrm{~mL}$ of trypanosome dilution buffer (TDB) $(5 \mathrm{mM} \mathrm{KCl}, 80 \mathrm{mM} \mathrm{NaCl}$, $1 \mathrm{mM} \mathrm{MgSO}$, $20 \mathrm{mM} \mathrm{Na}_{2} \mathrm{HPO}_{4}, 2 \mathrm{mM} \mathrm{NaH} \mathrm{PO}_{4}, 20 \mathrm{mM}$ glucose, $\mathrm{pH}$ 7.4) containing $10 \mu \mathrm{g}$ of NbAn33-PLGA NPs. Samples were washed and resuspended in $0.5 \mathrm{~mL}$ of cold PBS. Parasites were fixed ( $24 \mathrm{~h}$, $4{ }^{\circ} \mathrm{C}$ ) in $2.5 \%$ glutaraldehyde in a $0.1 \mathrm{M}$ sodium cacodylate buffer ( $\mathrm{pH} 7.2$ ) inside a humid chamber, and then mounted onto poly-Llysine slides $\left(24 \mathrm{~h}, 4^{\circ} \mathrm{C}\right)$. Sample post fixation was performed in $2 \%$ osmium tetroxide ( $1 \mathrm{~h}$, room temperature). Then, samples were dehydrated by incubating in $50 \%, 70 \%, 90 \%$, and $100 \%$ ethanol concentrations (two changes of $15 \mathrm{~min}$ in each) at room temperature.
Critical point drying was performed in a Leica EM CPD300 critical point dryer (Leica Microsystems GmbH, Wetzlar Germany). Samples were mounted on stubs for field emission scanning electron microscopy (FeSEM) and metalized in the presence of carbon in a Leica EM CED030 carbon thread evaporator (Leica Microsystems GmbH, Wetzlar Germany). Parasites were observed in an AURIGA dual beamfocused ion beam (FIB)-FeSEM workstation (Carl Zeiss Microscopy $\mathrm{GmbH}$, Jena, Germany).

\subsection{Nanoparticle uptake}

Monomorphic bloodstream forms of $T$. brucei were incubated $\left(10 \mathrm{~min}, 37^{\circ} \mathrm{C}\right.$ ) in TDB with fluorescein-labelled NbAn33-PLGA NPs. NP excess was removed by centrifugation. Then, the parasites were re-suspended in TDB containing the tomato lectin-Alexa Fluor ${ }^{\circledR} 594$ conjugate (a specific marker of the endocytic pathway in T. brucei [47]), and incubation ended after $10 \min \left(37^{\circ} \mathrm{C}\right)$ before fixation. Finally, the cells were fixed in $4 \%$ paraformaldehyde (PFA) in PBS, and they were then observed with a confocal laser-scanning microscope LSM 710 (Carl Zeiss Microscopy GmbH, Jena, Germany). Analysis of the images was done with a ZEN 2012 imaging software (Carl Zeiss Microscopy GmbH, Jena, Germany).

\subsection{Ethics statement}

The experiments, maintenance and care of mice complied with the guidelines of the European Convention for the Protection of Vertebrate Animals used for Experimental and other Scientific Purposes (CETS $\mathrm{n}^{\circ}$ 123). In vivo experiments were approved by the Ethical Committee for Animal Experiments of the Institute of Parasitology and Biomedicine "López-Neyra" (CSIC, Granada, Spain).

\section{Results}

\subsection{NP design and stability}

The drug carrier was designed for active targeting of the trypanocidal drug pentamidine to the T. brucei surface, as indicated in Fig. 1. PLGA NPs were synthesized by a water-in-oil-in-water (w/o/w) double emulsion/solvent evaporation (DE/SEV) technique [43-45], which allowed the formation of well-stabilized particles with spherical morphology and a narrow colloidal size distribution suitable for parenteral administration (average diameter, $145 \mathrm{~nm}$, Table 1). Particle size is a critical parameter for the treatment of trypanosomiasis. NPs should have an appropriate size to access the flagellar pocket, the unique location for endocytosis, through a narrow aperture between the flagellum and the membrane invagination [48]. Finally, PEG coating density of PLGA NPs was $18 \%$ ( $\mathrm{mg}$ of PEG at the surface for $100 \mathrm{mg}$ of particles).

To specifically target trypanosomes pentamidine-PLGA NPs were coupled to nanobody NbAn33, which specifically recognizes a conserved $\mathrm{N}$-linked Man 5-9 (oligomannose) carbohydrate epitope that is present in the GPI anchor of the parasite surface protein (Fig. 1A) [41]. UV-Vis spectrophotometric measurements were 
used to quantify the density of NbAn33 bound to PLGA NPs. The amount of NbAn33 conjugated to the NPs was $0.08 \mathrm{mg}$ of nanobody per mg of NPs. Heterofuntional $\mathrm{PEG}_{3} \mathrm{KDa}, \mathrm{NH}_{2}-\mathrm{PEG}-\mathrm{COOH}$, chains was employed to link NbAn33 to PLGA NPs (Fig. 1B). The molecular weight of the PEG, $3 \mathrm{kDa}$, was an important parameter in the design of this nanocarrier. Its chain length should be long enough to allow the nanobody to reach its recognition epitope concealed within the densely packed VSG surface coat (Fig. 1). Finally, particle size and the quality of the nanoparticulate suspensions did not change significantly when the particles were loaded with different quantities of the trypanocidal drug (Table 1). Both, the small size and PEG coating prolong the circulation time of the NPs, thus enhancing their parasite targeting capacity in the infected host $[49,50]$.

\subsection{Pentamidine absorption into NbAn33-PLGA NPS}

Table 1 shows the amount of trypanocidal drug absorbed by the NPs as a function of the pentamidine concentration. It can be observed how the drug concentration positively influenced the absorption efficiency into the NPs. For instance, pentamidine entrapment efficiency (\%) and pentamidine loading (\%) values rise from $12 \%$ and $0.0005 \%$ (when the drug concentration was $10^{-5} \mathrm{M}$ ) to $72 \%$ and $3 \%$ (when the drug concentration was $10^{-2} \mathrm{M}$ ), respectively. Remarkably, the production performance (\%) in all the formulations conditions was always $\geq 93 \%$. Thus, in vitro and in vivo studies were performed using the Nps with the greater pentamidine entrapment efficiencies, i.e., 72\% (Table 1).

The electrokinetic characterization of the NPs highlighted the similarity between the zeta potential, $\zeta(\mathrm{mV})$ values of PLGA NPs and pentamidine-PLGA NPs (Table 1 ). These results demonstrated that pentamidine molecules were efficiently encapsulated into the NPs rather than just adsorbed at the surface.

\subsection{Pentamidine release from NbAn33-pentamidine-PLGA NPS}

Drug release from NbAn33-pentamidine-PLGA NPs was analyzed at pH 7.4 (physiological pH) and pH 5 (lysosomal pH). Pentamidine release was pH dependent and followed a biphasic profile (Fig. 3), characterized by an initial fast (burst) release (up to $45 \%$ in $12 \mathrm{~h}$ at $\mathrm{pH} 7.4$, and up to $35 \%$ in $1 \mathrm{~h}$ at $\mathrm{pH} 5$ ) followed by a much slower release phase (over a period of 11.5 days at $\mathrm{pH} 7.4$, and over a period of $23 \mathrm{~h}$ at $\mathrm{pH} 5$ ). Such pentamidine release profile confirmed that the major fraction of the drug was entrapped into the nanoparticle rather adsorbed onto the particle surface. In addition, this release profile could be due to diffusion-cum-degradation mediated processes. The first phase may result from drug diffusion through the external side of the nanoparticle [51-53]. The second pentamidine release phase could be the consequence of drug diffusion through the nanoparticle structure, nanoparticle degradation, or both [52-55].

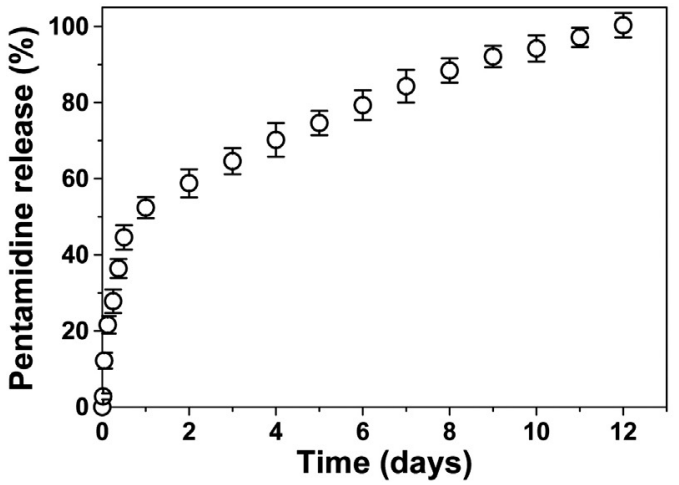

Finally, it can be assumed that pentamidine loading into the PLGA NPs was kept unaltered during surface functionalization with NbAn33, given the negligible drug release $(<3 \%)$ from the NPs.

\subsection{In vitro cytotoxicity studies}

The effectiveness of different pentamidine formulations was evaluated in vitro using the resarzurin assay (Fig. 4). The halfinhibitory concentration $\left(\mathrm{IC}_{50}\right)$ of free pentamidine was $9.58 \pm$ 0.27 nM (Fig. 4 A, B). Pentamidine loaded within PLGA NPs was significantly more efficient at killing trypanosomes. As shown in Fig. $4 \mathrm{~B}$, there was a 3 -fold reduction in the $\mathrm{IC}_{50}$ of pentamidine-PLGA NPs relative to free drug. Finally, the $\mathrm{IC}_{50}$ of NbAn33-pentamidinePLGA NPs was $1.37 \pm 0.10 \mathrm{nM}$, which represented a 7-fold reduction in the $\mathrm{IC}_{50}$ relative to free drug (Fig. $4 \mathrm{~B}$ ). Thus, active targeting with the nanobody yielded an additional 4 -fold reduction of the $\mathrm{IC}_{50}$ relative to passive targeting, emphasizing the importance of the nanobody coating in the effectiveness of the nanocarrier.

Several in vitro studies have described drug delivery systems based on PLGA NPs for the passive targeting of protozoan parasite infections, mainly Leishmaniasis, Malaria and Chagas disease [56-59]. In all cases, only small decreases in $\mathrm{IC}_{50}$ relative to free drugs were obtained. Therefore, our results highlight the importance of active targeting to treat infectious protozoan diseases.

\subsection{In vivo therapy studies}

We next assayed the efficacy of NbAn33-pentamidine-PLGA NPs in vivo in an acute murine model of African trypanosomiasis. The minimal full curative dose of free pentamidine is a daily intraperitoneally dose of $2.5 \mathrm{mg} \mathrm{kg}^{-1}$ for four days $[60,61]$. Therapeutic treatments began on the 3rd day post-infection and untreated animals died at day 5 (Fig. 5). Mice were also treated with free pentamidine and NbAn33pentamidine-PLGA NPs at 10 - and 100-fold lower doses than the minimal full curative dose, 0.25 and $0.025 \mathrm{mg} \mathrm{kg}^{-1}$, respectively (Fig. 5).

NbAn33-pentamidine-PLGA NPs cured all infected mice at a 10-fold lower dose $\left(0.25 \mathrm{mg} \mathrm{kg}^{-1}\right)$. At the same dose, all mice treated with free pentamidine died between days 24 and 27 post infection. At a 100-fold lower dose, the NbAn33-pentamidine-PLGA NPs formulation cured 60\% of mice, while free pentamidine failed to cure any of the treated mice, killing all animals between days 5 and 19 after infection. Pentamidine-PLGA NPs (without nanobody coating) at a 10 -fold dilution $\left(0.25 \mathrm{mg} \mathrm{kg}^{-1}\right)$ cured $40 \%$ of treated mice, while at a 100 -fold dilution $\left(0.025 \mathrm{mg} \mathrm{kg}^{-1}\right)$ all mice died between days 18 and 23 postinfection.

According to these results, our system seems to be more effective in vivo than in vitro, with at least a 10 -fold reduction of the full curative dose relative to free drug. These results clearly demonstrate the decisive

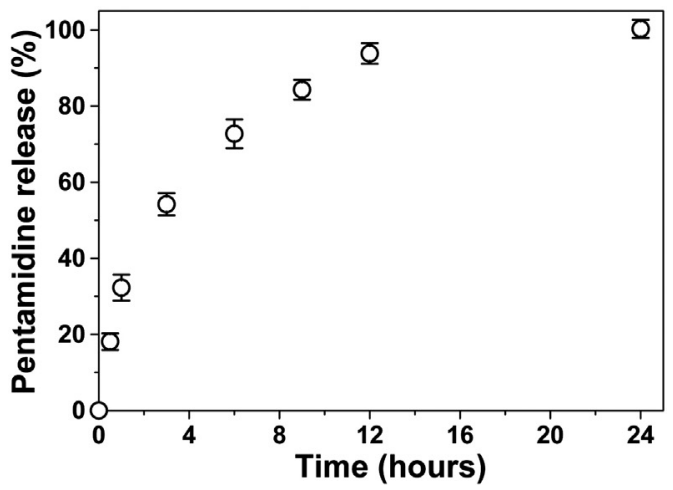

Fig. 3. Release of entrapped pentamidine from NbAn33-pentamidine-PLGA NPs as a function of the incubation time in PBS pH 7.4 (right panel) and pH 5.5 (left panel) at $37{ }^{\circ} \mathrm{C}$. 
A

- NbAn33-Pentamidine-PLGA NPs

- Pentamidine-PLGA NPs

- Pentamidine

- PLGA NPs

- NbAn33-PLga nPs



Log [Pentamidine] (M)
B



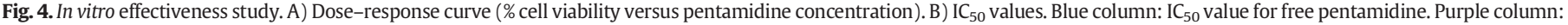
$\mathrm{IC}_{50}$ value for pentamidine-PLGA NPs. Green column: $\mathrm{IC}_{50}$ value for NbAn33-Pentamidine-PLGA NPs. Errors bars indicate SD from 3-9 independent experiments.

contribution of nanobody conjugation in the effectiveness of the drug delivery system.

\subsection{NbAn33-pentamidine-PLGA NPs bind to trypanosome surface and are endocytosed}

The binding capacity of NbAn33-pentamidine-PLGA NPs to the parasite surface was assessed by FESEM (Fig. 6). Spherical NPs of different sizes were clearly observed adhered to the trypanosome surface (Fig. 6b). As mentioned above, NPs size is a critical parameter as they have to cross a small aperture to access the flagellar pocket (Fig. 6c).

Finally, to visualise drug entrance and localisation NbAn33-PLGA NPs labelled with fluorescein were incubated with live trypanosome in the presence of Alexa 594-tomato lectin, a specific marker of the endocytic pathway in T. brucei [47]. As expected, labelled NbAn33PLGA NPs were detected on the surface of the parasite being internalized and accumulating in the endocytic pathway (Fig. 7). Pentamidine needs to reach high intracellular concentrations for effective activity [62]. Thus, the nanocarrier concentrates NPs drug cargo via the endocytosis pathway into the cytoplasm, increasing the efficacy of the drug.

\section{Discussion}

This investigation is devoted to the development of a new drug delivery system for the active targeting of pentamidine toward African trypanosomes. Concretely, the nanoplatform is based on PLGA NPs functionalized to obtain passive drug targeting capabilities (PEGylation for extended plasma circulation times) and active drug targeting capabilities (surface decoration with NbAn33 moieties that can specifically recognize cryptic epitopes onto the surface of $T$. brucei). NP synthesis was based on a reproducible w/o/w DE/SEV methodology, and NP functionalization with PEG chains and nanobody moieties relied on a standard EDC/NHS chemistry.

Under the best formulation conditions, well-stabilized spherical NPs were obtained, suitable for the parenteral route of administration (average diameter $\approx 145 \mathrm{~nm}$, Table 1 ). Both, the small size and PEG coating may contribute to the extended blood circulation times, thus facilitating the active drug targeting to the parasite [49,50]. Even more, such properties are specially relevant, given the fact that the NPs should have an appropriate size to access the flagellar pocket, the unique location where endocytosis occurs at the narrow aperture between the flagellum and the membrane invagination [48]. In addition, the length of the PEG chains $\left(\mathrm{M}_{\mathrm{w}}: 3 \mathrm{kDa}\right)$ was enough to allow the nanobody to reach the recognition epitope located within the densely packed VSG (Fig. 1A).

Pentamidine loading procedure yielded significant drug entrapment efficiencies and sustained drug release properties (Table 1, Fig. 3 ). In addition, NP composition determined a $\mathrm{pH}$-responsive drug release which could be the consequence of the specific polymer breakage in acidic environments (those existing within the lysosomes of the parasite). Such drug release behaviour can be considered advantageous for intracellular pentamidine delivery.

Regarding the preclinical tests, pentamidine activity in vitro against trypanosomes was significantly optimized when the drug was incorporated within the nanoparticulate formulation. Concretely, 7 -fold reduction was obtained in the $\mathrm{IC}_{50}$ values relative to the free drug, and also an additional 4 -fold reduction in the $\mathrm{IC}_{50}$ levels relative to the PEGylated nanoformulation (Fig. 4), this emphasizing the critical

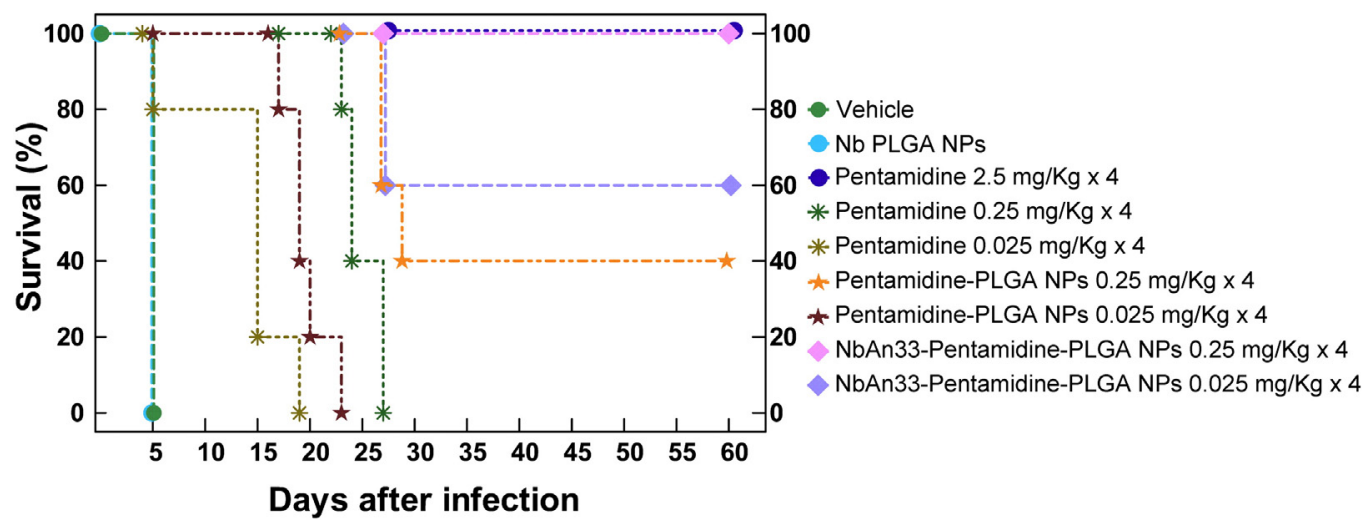

Fig. 5. In vivo therapy experiments. Survival (Kaplan-Meier plot) of female C57BL/6J mice infected with T. brucei AnTat1.1 (104 parasites). Vehicle; physiological serum. 

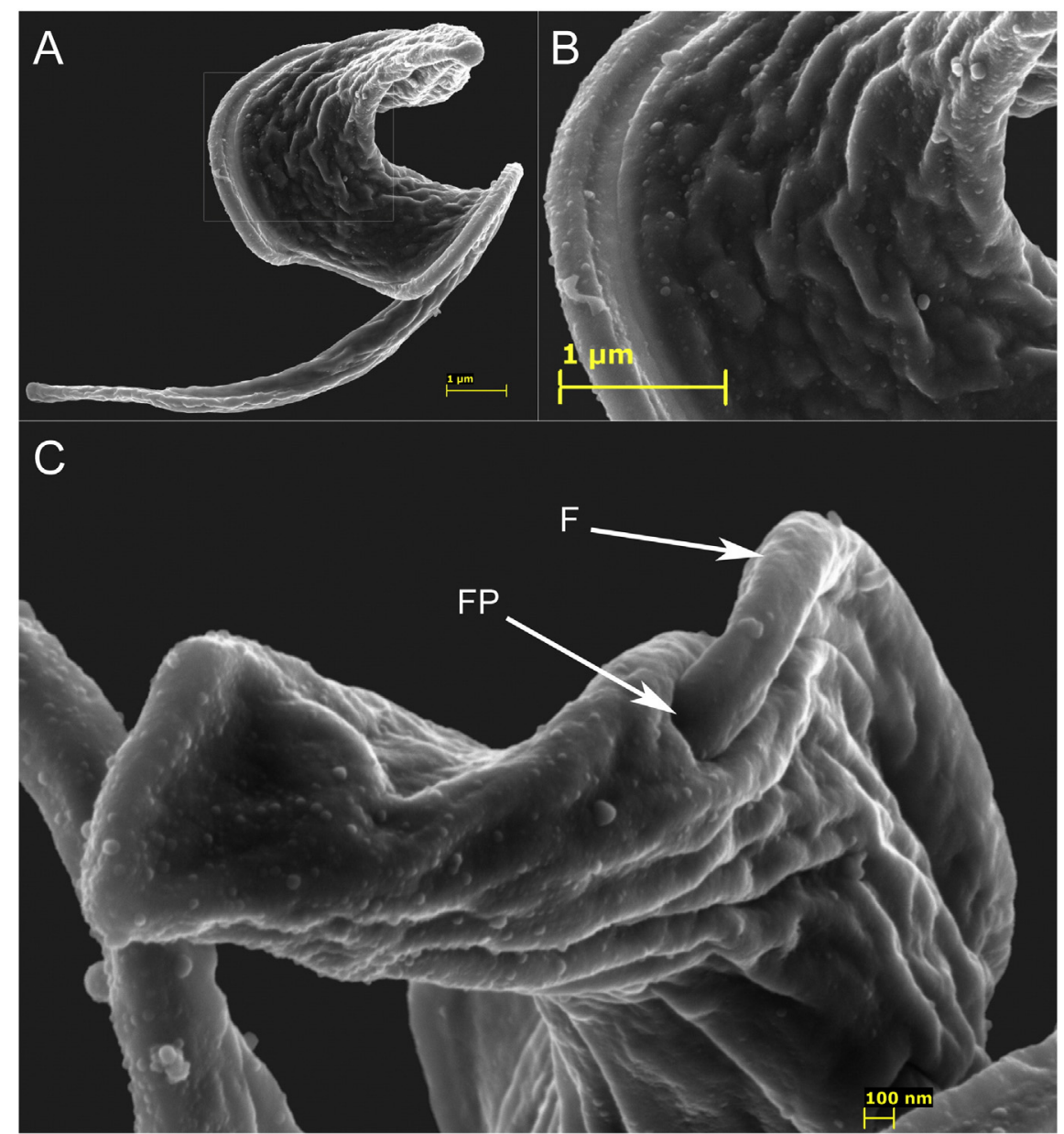

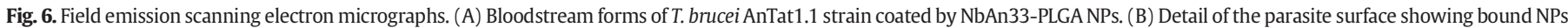
(C) A view of the flagellar pocket aperture with NPs in the process of being endocytosed. FP, flagellar pocket aperture; F, flagellum.

role displayed by the NbAn33 surface functionalization. This more effective activity of pentamidine when loaded to the nanoparticulate system surface functionalized with NbAn33 molecules was confirmed in vivo (Fig. 5): it was determined at least a 10 -fold reduction in the

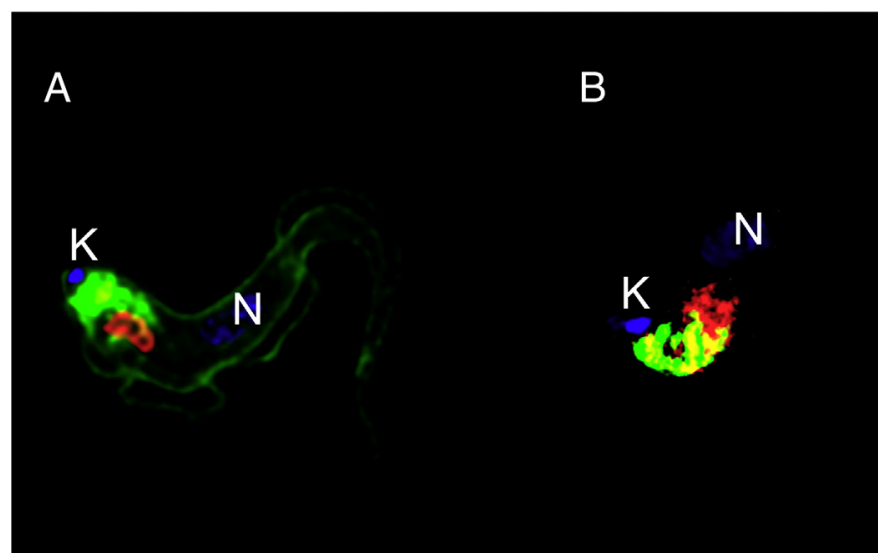

Fig. 7. NbAn33-pentamidine-PLGA NPs internalized and accumulated in the endocytic pathway. A) Live monomorphic bloodstream forms of T. brucei AnTat1.1 were incubated with fluorescein labelled NbAn33-PLGA NPs (green) for 10 min at $37^{\circ} \mathrm{C}$. NP excess was removed and the parasites were incubated with tomato lectin-Alexa Fluor 594 conjugate. Samples were taken after $1 \mathrm{~min}(\mathrm{~A})$ and $10 \mathrm{~min}(\mathrm{~B})$ of incubation. Nuclei and kinetoplasts (mitochondrial DNA) were stained with DAPI, in blue. N, nuclei. K, kinetoplast. (For interpretation of the references to colour in this figure legend, the reader is referred to the web version of this article.) minimum full curative dose relative to the free drug. Such optimized cytotoxic activity may contribute to the reduction of the pentamidine doses needed to assure a therapeutic effect and, subsequently, to the minimization of the adverse side effects associated to the mechanism of action of the drug.

Finally, an additional proof-of-concept was done to investigate the binding capacity of NbAn33-PLGA NPs to the parasite surface. FeSEM observations demonstrated the interaction of the NPs with the flagellar pocket (Fig. 6), a key step prior to particle uptake by endocytosis [48]. In addition, confocal microscopy analysis confirmed the particle uptake/internalization by endocytosis (Fig. 7). Thus, the drug (loaded to the NPs) may satisfactorily enter into the cytoplasm of the trypanosome via the endocytic pathway, leading to the highly enough intracellular drug concentrations that will display the efficient cytotoxic activity [62].

\section{Conclusions}

This study demonstrates that the trypanosome surface is an excellent therapeutic target due to the high turnover of the entire cell membrane. The small size of nanobodies allows them to reach conserved epitopes located in locations which are inaccessible to conventional antibodies. Nanobody conjugation was essential for the effectiveness of the formulations in both in vitro and in vivo experiments. Our system employs components approved for their use in humans, including nanobodies, and may be loaded with drugs that are currently in use for HAT, making them ready for their practical use in therapy. Thus, this effective and flexible targeted drug delivery system represents 
an alternative therapeutic approach to the actual anti-trypanosome therapies and it could be easily adapted to treat other diseases.

\section{Acknowledgements}

The authors acknowledge founding from the Plan Nacional de Investigación (Ministerio de Economía y Competitividad, Spain) grant SAF2011-30528, European Union grant FP7-HEALTH-2007-B2.3.4-1.223048 (Nanotryp) and from the Instituto de Salud Carlos III, Spain, grant FIS 11/02571. We thank to Estela Pineda Molina (LECCSIC, Granada) for technical advice in protein purification. We also thank to the electron microscopy service and the biological processing laboratory of the Scientific Instrumentation Center (University of Granada, Granada, Spain) for FESEM sample processing and observation.

\section{References}

[1] M.P. Barrett, R.J. Burchmore, A. Stich, J.O. Lazzari, A.C. Frasch, J.J. Cazzulo, S. Krishna, The trypanosomiases, Lancet 362 (2003) 1469-1480.

[2] R. Brun, J. Blum, Human African trypanosomiasis, Infect. Dis. Clin. N. Am. 26 (2012) 261-273.

[3] R. Brun, J. Blum, F. Chappuis, C. Burri, Human African trypanosomiasis, Lancet 375 (2010) 148-159.

[4] M.P. Barrett, The fall and rise of sleeping sickness, Lancet 353 (1999) 1113-1114

[5] M.P. Barrett, The rise and fall of sleeping sickness, Lancet 367 (2006) 1377-1378.

[6] D. Horn, R. McCulloch, Molecular mechanisms underlying the control of antigenic variation in African trypanosomes, Curr. Opin. Microbiol. 13 (2010) 700-705.

[7] G. Rudenko, African trypanosomes: the genome and adaptations for immune evasion, Essays Biochem. 51 (2011) 47-62.

[8] P. Overath, M. Engstler, Endocytosis, membrane recycling and sorting of GPIanchored proteins: Trypanosoma brucei as a model system, Mol. Microbiol. 53 (2004) 735-744

[9] C. O'Beirne, C.M. Lowry, H.P. Voorheis, Both IgM and IgG anti-VSG antibodies initiate a cycle of aggregation-disaggregation of bloodstream forms of Trypanosoma brucei without damage to the parasite, Mol. Biochem. Parasitol. 91 (1998) 165-193.

[10] M. Engstler, T. Pfohl, S. Herminghaus, M. Boshart, G. Wiegertjes, N. Heddergott, P. Overath, Hydrodynamic flow-mediated protein sorting on the cell surface of trypanosomes, Cell 131 (2007) 505-515.

[11] D. Salmon, M. Geuskens, F. Hanocq, J. Hanocq-Quertier, D. Nolan, L. Ruben, E. Pays, A novel heterodimeric transferrin receptor encoded by a pair of VSC expression site-associated genes in T. brucei, Cell 78 (1994) 75-86.

[12] D. Steverding, Y.D. Stierhof, H. Fuchs, R. Tauber, P. Overath, Transferrin-binding protein complex is the receptor for transferrin uptake in Trypanosoma brucei, J. Cell Biol. 131 (1995) 1173-1182

[13] A. Luscher, H.P. de Koning, P. Maser, Chemotherapeutic strategies against Trypanosoma brucei: drug targets vs. drug targeting, Curr. Pharm. Des. 13 (2007) 555-567.

[14] J. Garcia-Salcedo, J. Munday, J. Unciti-Broceta, H. Koning, Progress towards new treatments for human African trypanosomiasis, in: S. Magez, M. Radwanska (Eds.) Trypanosomes and Trypanosomiasis, Springer, Vienna, 2014, pp. 217-238.

[15] O. Kayser, C. Olbrich, S.L. Croft, A.F. Kiderlen, Formulation and biopharmaceutical issues in the development of drug delivery systems for antiparasitic drugs, Parasitol Res. 90 (Suppl. 2) (2003) S63-S70.

[16] S.M. Moghimi, D. Peer, R. Langer, Reshaping the future of nanopharmaceuticals: ad iudicium, ACS Nano 5 (2011) 8454-8458.

[17] S. Mitragotri, J. Lahann, Materials for drug delivery: innovative solutions to address complex biological hurdles, Adv. Mater. 24 (2012) 3717-3723.

[18] A. Beletsi, Z. Panagi, K. Avgoustakis, Biodistribution properties of nanoparticles based on mixtures of PLGA with PLGA-PEG diblock copolymers, Int. J. Pharm. 298 (2005) 233-241.

[19] T. Betancourt, J.D. Byrne, N. Sunaryo, S.W. Crowder, M. Kadapakkam, S. Patel, S Casciato, L. Brannon-Peppas, PEGylation strategies for active targeting of PLA/PLGA nanoparticles, J. Biomed. Mater. Res. A 91 (2009) 263-276.

[20] F. Danhier, E. Ansorena, J.M. Silva, R. Coco, A. Le Breton, V. Préat, PLGA-based nanoparticles: an overview of biomedical applications, J. Control. Release 161 (2012) 505-522.

[21] J.P. Bertram, S.M. Jay, S.R. Hynes, R. Robinson, J.M. Criscione, E.B. Lavik, Functionalized poly(lactic-co-glycolic acid) enhances drug delivery and provides chemical moieties for surface engineering while preserving biocompatibility, Acta Biomater. 5 (2009) 2860-2871.

[22] S. Fredenberg, M. Wahlgren, M. Reslow, A. Axelsson, The mechanisms of drug release in poly(lactic-co-glycolic acid)-based drug delivery systems-a review, Int. J. Pharm. 415 (2011) 34-52.

[23] D. Klose, F. Siepmann, K. Elkharraz, J. Siepmann, PLGA-based drug delivery systems: importance of the type of drug and device geometry, Int. J. Pharm. 354 (2008) 95-103.

[24] V.K. Nguyen, A. Desmyter, S. Muyldermans, Functional heavy-chain antibodies in Camelidae, Adv. Immunol. 79 (2001) 261-296.

[25] S. Muyldermans, C. Cambillau, L. Wyns, Recognition of antigens by single-domain antibody fragments: the superfluous luxury of paired domains, Trends Biochem. Sci. 26 (2001) 230-235.
[26] K. Els Conrath, M. Lauwereys, L. Wyns, S. Muyldermans, Camel single-domain antibodies as modular building units in bispecific and bivalent antibody constructs, J. Biol. Chem. 276 (2001) 7346-7350.

[27] C. Hamers-Casterman, T. Atarhouch, S. Muyldermans, G. Robinson, C. Hamers, E.B. Songa, N. Bendahman, R. Hamers, Naturally occurring antibodies devoid of light chains, Nature 363 (1993) 446-448.

[28] V.K. Nguyen, R. Hamers, L. Wyns, S. Muyldermans, Loss of splice consensus signal is responsible for the removal of the entire $\mathrm{C}(\mathrm{H}) 1$ domain of the functional camel IGG2A heavy-chain antibodies, Mol. Immunol. 36 (1999) 515-524.

[29] K.B. Vu, M.A. Ghahroudi, L. Wyns, S. Muyldermans, Comparison of llama VH sequences from conventional and heavy chain antibodies, Mol. Immunol. 34 (1997) 1121-1131.

[30] S. Muyldermans, T. Atarhouch, J. Saldanha, J.A. Barbosa, R. Hamers, Sequence and structure of $\mathrm{VH}$ domain from naturally occurring camel heavy chain immunoglobulins lacking light chains, Protein Eng. 7 (1994) 1129-1135.

[31] J.D. Unciti-Broceta, T. Del Castillo, M. Soriano, S. Magez, J.A. Garcia-Salcedo, Novel therapy based on camelid nanobodies, Ther. Deliv. 4 (2013) 1321-1336.

[32] P. Vanlandschoot, C. Stortelers, E. Beirnaert, L.I. Ibanez, B. Schepens, E. Depla, X. Saelens, Nanobodies(R): new ammunition to battle viruses, Antivir. Res. 92 (2011) 389-407.

[33] S. Oliveira, R.M. Schiffelers, J. van der Veeken, R. van der Meel, R. Vongpromek, P.M. van Bergen En Henegouwen, G. Storm, R.C. Roovers, Downregulation of EGFR by a novel multivalent nanobody-liposome platform, J. Control. Release 145 (2010) 165-175.

[34] T.N. Baral, S. Magez, B. Stijlemans, K. Conrath, B. Vanhollebeke, E. Pays, S. Muyldermans, P. De Baetselier, Experimental therapy of African trypanosomiasis with a nanobody-conjugated human trypanolytic factor, Nat. Med. 12 (2006) 580-584.

[35] A. Järviluoma, T. Strandin, S. Lülf, J. Bouchet, A.R. Mäkelä, M. Geyer, S. Benichou, K. Saksela, High-affinity target binding engineered via fusion of a single-domain antibody fragment with a ligand-tailored SH3 domain, PLoS One 7 (2012) e40331.

[36] M. Behdani, S. Zeinali, M. Karimipour, H. Khanahmad, S. Schoonooghe, A. Aslemarz, N. Seyed, R. Moazami-Godarzi, F. Baniahmad, M. Habibi-Anbouhi, G. HassanzadehGhassabeh, S. Muyldermans, Development of VEGFR2-specific Nanobody Pseudomonas exotoxin A conjugated to provide efficient inhibition of tumor cell growth, N. Biotechnol. 30 (2013) 205-209.

[37] B. Van De Broek, N. Devoogdt, A. Dhollander, H.L. Gijs, K. Jans, L. Lagae, S. Muyldermans, G. Maes, G. Borghs, Specific cell targeting with nanobody conjugated branched gold nanoparticles for photothermal therapy, ACS Nano 5 (2011) 4319-4328.

[38] R. van der Meel, S. Oliveira, I. Altintas, R. Haselberg, J. van der Veeken, R.C. Roovers, P.M. van Bergen en Henegouwen, G. Storm, W.E. Hennink, R.M. Schiffelers, R.J. Kok, Tumor-targeted Nanobullets: Anti-EGFR nanobody-liposomes loaded with anti-IGF-1R kinase inhibitor for cancer treatment, J. Control. Release 159 (2012) 281-289.

[39] I. Altintas, R. Heukers, R. van der Meel, M. Lacombe, M. Amidi, P.M.P. van Bergen en Henegouwen, W.E. Hennink, R.M. Schiffelers, R.J. Kok, Nanobody-albumin nanoparticles (NANAPs) for the delivery of a multikinase inhibitor 17864 to EGFR overexpressing tumor cells, J. Control. Release 165 (2013) 110-118.

[40] D. Smolarek, C. Hattab, G. Hassanzadeh-Ghassabeh, S. Cochet, C. Gutierrez, A.G. de Brevern, R. Udomsangpetch, J. Picot, M. Grodecka, K. Wasniowska, S. Muyldermans, Y. Colin, C. Le Van Kim, M. Czerwinski, O. Bertrand, A recombinant dromedary antibody fragment (VHH or nanobody) directed against human Duffy antigen receptor for chemokines, Cell. Mol. Life Sci. 67 (2010) 3371-3387.

[41] B. Stijlemans, K. Conrath, V. Cortez-Retamozo, H. Van Xong, L. Wyns, P. Senter, H. Revets, P. De Baetselier, S. Muyldermans, S. Magez, Efficient targeting of conserved cryptic epitopes of infectious agents by single domain antibodies. African trypanosomes as paradigm, J. Biol. Chem 279 (2004) 1256-1261.

[42] K.E. Conrath, M. Lauwereys, M. Galleni, A. Matagne, J.M. Frere, J. Kinne, L. Wyns, S. Muyldermans, Beta-lactamase inhibitors derived from single-domain antibody fragments elicited in the camelidae, Antimicrob. Agents Chemother. 45 (2001) 2807-2812.

[43] R.A. Jain, The manufacturing techniques of various drug loaded biodegradable poly(lactide-co-glycolide) (PLGA) devices, Biomaterials. 21 (2000) 2475-2490.

[44] M.J. Cózar-Bernal, M.A. Holgado, J.L. Arias, I. Muñoz-Rubio, L. Martín-Banderas, J. Álvarez-Fuentes, M. Fernández-Arévalo, Insulin-loaded PLGA microparticles: flow focusing versus double emulsion/solvent evaporation, J. Microencapsul. 28 (2011) 430-441.

[45] B. Pérez-Artacho, V. Gallardo, M.A. Ruiz, M.A. Ruiz, J. Arias, Maghemite/poly(d,llactide-co-glycolyde) composite nanoplatform for therapeutic applications, J. Nanopart. Res. 14 (2012) 1-10.

[46] J.D. Unciti-Broceta, J. Maceira, S. Morales, A. Garcia-Perez, M.E. Munoz-Torres, J. A. Garcia-Salcedo, Nicotinamide inhibits the lysosomal cathepsin B-like protease and kills African trypanosomes, J. Biol. Chem. 288 (2013) 10548-10557.

[47] D.P. Nolan, M. Geuskens, E. Pays, N-linked glycans containing linear poly-Nacetyllactosamine as sorting signals in endocytosis in Trypanosoma brucei, Curr. Biol. 9 (1999) 1169-1172.

[48] M.C. Field, M. Carrington, The trypanosome flagellar pocket, Nat. Rev. Microbiol 7 (2009) 775-786.

[49] Q. Sun, M. Radosz, Y. Shen, Challenges in design of translational nanocarriers, J. Control. Release 164 (2012) 156-169.

[50] R. Gref, A. Domb, P. Quellec, T. Blunk, R.H. Müller, J.M. Verbavatz, R. Langer, The controlled intravenous delivery of drugs using PEG-coated sterically stabilized nanospheres, Adv. Drug Deliv. Rev. 64 (Supplement) (2012) 316-326.

[51] M.A. Ibrahim, A. Ismail, M.I. Fetouh, A. Göpferich, Stability of insulin during the erosion of poly(lactic acid) and poly(lactic-co-glycolic acid) microspheres, J. Controlled Rel. 106 (2005) 241-252. 
[52] M.A. Holgado, J. Álvarez-Fuentes, M. Fernández-Arévalo, J.L. Arias, Possibilities of Poly(D,L-lactide-co-glycolide) in the formulation of nanomedicines against cancer, Curr. Drug Targets 12 (2011) 1096-1111.

[53] L. Martín-Banderas, E. Sáez-Fernández, M.Á. Holgado, M.M. Durán-Lobato, J.C. Prados, C. Melguizo, J.L. Arias, Biocompatible gemcitabine-based nanomedicine engineered by Flow Focusing® for efficient antitumor activity, Int. J. Pharm. 443 (2013) 103-109.

[54] M.A. Holgado, J.L. Arias, M.J. Cózar, J. Alvarez-Fuentes, A.M. Gañán-Calvo, M. Fernández-Arévalo, Synthesis of lidocaine-loaded PLGA microparticles by flow focusing: Effects on drug loading and release properties, Int. J. Pharm. 358 (2008) 27-35.

[55] G. Mittal, D.K. Sahana, V. Bhardwaj, M.N.V. Ravi Kumar, Estradiol loaded PLGA nanoparticles for oral administration: Effect of polymer molecular weight and copolymer composition on release behavior in vitro and in vivo, J. Controlled Rel. 119 (2007) 77-85.

[56] S. Costa Lima, V. Rodrigues, J. Garrido, F. Borges, P. Kong Thoo Lin, A. Cordeiro da Silva, In vitro evaluation of bisnaphthalimidopropyl derivatives loaded into pegylated nanoparticles against Leishmania infantum protozoa, Int. J. Antimicrob. Agents. 39 (2012) 424-430.

[57] H. Van de Ven, M. Vermeersch, A. Matheeussen, J. Vandervoort, W. Weyenberg, S Apers, P. Cos, L. Maes, A. Ludwig, PLGA nanoparticles loaded with the antileishmanial saponin $\beta$-aescin: Factor influence study and in vitro efficacy evaluation, Int. J. Pharm. 420 (2011) 122-132.

[58] E.L. Romero, M.J. Morilla, Nanotechnological approaches against Chagas disease, Adv. Drug Deliv. Rev. 62 (2010) 576-588.

[59] N.S. Santos-Magalhães, V.C.F. Mosqueira, Nanotechnology applied to the treatment of malaria, Adv. Drug Deliv. Rev. 62 (2010) 560-575.

[60] J.K. Thuita, S.M. Karanja, T. Wenzler, R.E. Mdachi, J.M. Ngotho, J.M. Kagira, R. Tidwell, R. Brun, Efficacy of the diamidine DB75 and its prodrug DB289, against murine models of human African trypanosomiasis, Acta Trop. 108 (2008) 6-10.

[61] J.A. Frearson, S. Brand, S.P. McElroy, L.A. Cleghorn, O. Smid, L. Stojanovski, H.P. Price, M.L. Guther, L.S. Torrie, D.A. Robinson, I. Hallyburton, C.P. Mpamhanga, J.A. Brannigan, A.J. Wilkinson, M. Hodgkinson, R. Hui, W. Qiu, O.G. Raimi, D.M. van Aalten, R. Brenk, I.H. Gilbert, K.D. Read, A.H. Fairlamb, M.A. Ferguson, D.F. Smith, P. G. Wyatt, N-myristoyltransferase inhibitors as new leads to treat sleeping sickness, Nature 464 (2010) 728-732.

[62] D. Damper, C.L. Patton, Pentamidine transport and sensitivity in brucei-group trypanosomes, J. Protozool. 23 (1976) 349-356. 